\title{
Utilizando a Rede Neural RBF Como Mecanismo Auxiliador no Diagnóstico da Doença da Murcha Fitóftora em Pimentões
}

\author{
Roney Nogueira de Sousa ${ }^{1}$, Lucas José Lemos Braz ${ }^{2}$, \\ Janaide Nogueira de Sousa Ximenes ${ }^{3}$, Rhyan Ximenes de Brito ${ }^{1}$ \\ ${ }^{1}$ Instituto Federal de Educação, Ciência e Tecnologia do Ceará (IFCE) \\ CE-187, s/n - Estádio, - CEP 62320-000 - Tianguá - CE - Brasil \\ ${ }^{2}$ Faculdade IEducare (FIED) - Rua Conselheiro João Lourenço, \\ 406 - CEP 62320-000 - Tianguá - CE - Brasil \\ ${ }^{3}$ Universidade Federal do Ceará (UFC) - Rua Coronel Estanislau \\ Frota 553 - Centro - CEP 62010-560 - Sobral - CE - Brasil \\ \{nogueiraroney453, lucas.jbl19, nogueirajanaide, rxbrio\}@gmail.com
}

\begin{abstract}
The use of Artificial Intelligence techniques has stood out in solving several types of problems. Based on this, a study was carried out aiming to use the neural network Radial Basis Function (RBF) as a support tool in the prediagnosis of wilted phytophorous disease in a pepper garden. The methodology was implemented using a database, with emphasis on training and testing to classify the samples used. 10 training sessions were carried out with normalized and balanced data, the cross validation ( $k$-fold) was used. The results were analyzed statistically, obtaining an average rate of $93.37 \%$ of correct answers.
\end{abstract}

Resumo. A utilização de técnicas de Inteligência Artificial tem-se destacado na resolução de diversos tipos de problemáticas. Com base nisso, foi realizado um estudo objetivando utilizar a rede neural Radial Basis Function (RBF) como ferramenta de apoio no pré-diagnóstico da doença murcha de fitóftora em uma horta de pimentões. A metodologia foi implementada utilizando-se de uma base de dados, com ênfase no treinamento e teste para classificação das amostras utilizadas. Foram feitos 10 treinamentos com os dados normalizados e balanceados, foi utilizada a validação cruzada ( $k$-fold). Os resultados foram analisados estatisticamente obtendo-se uma taxa média de 93,3\% de acertos.

\section{Introdução}

O pimentão é uma das dez hortaliças de maior importância no Brasil. No Ceará, especificamente, seu valor deve-se não somente em razão da forte participação na culinária doméstica e empresarial, como também ao aspecto social que assume [Viana et al. 2007], no entanto, devido a doenças e pragas que afetam o plantio do fruto as mesmas geram impactantes danos na produção de pequenos e grandes agricultores.

O patógeno afeta primeiramente o sistema radicular e o colo da planta, podendo também atacar a parte aérea. A Phytophthora capsici Leonian (PCL) é um patógeno polífago, amplamente distribuído nos solos cultivados do Brasil e de muitos outros países, sendo de difícil controle considerada uma das doenças fúngicas mais importantes para a cultura do pimentão [Lopes and de Ávila 2005]. 
Nesses termos, faz-se necessário a utilização de técnicas computacionais que auxiliem no diagnóstico da doença. Tendo como parâmetros atributos como: necrose de folhas, manchas bronzeadas circulares, mofo branco e escurecimento do caule, entre outros auxiliam no diagnóstico precoce da doença em plantas do gênero.

Esse trabalho tem como principal objetivo realizar um estudo utilizando o algoritmo da rede neural $\mathrm{RBF}$ (Radial Basis Function) que estão disponíveis no aplicativo Waikato Environment for Knowledge Analysis (WEKA), com o objetivando auxiliar no diagnóstico da murcha de fitóftora. O estudo foi realizado a partir de um banco de dados construído com 127 amostras com 6 atributos.

A motivação do trabalho em questão deve-se a necessidade do aprimoramento de técnicas que garantam a identificação precoce do patógeno de forma que se possa tomar medidas que visem o manejo e controle da doença de modo a reduzir perdas na produção.

\section{Trabalhos Relacionados}

Esta seção traz resumos sobre diferentes abordagens do uso da Inteligência Artificial aplicada como ferramenta de auxílio no diagnóstico de doenças com plantas.

[Castro 2012] construiu um modelo completo em nível de árvore individual para fragmento de Floresta Semidecidual Montana utilizando redes neurais artificiais (RNA). Os dados foram provenientes de dez parcelas permanentes em um fragmento florestal de 17 hectares no município de Viçosa - MG em estágio médio de sucessão ecológica monitoradas durante 14 anos.

[Galvão et al. 2018] utilizou técnicas de visão computacional, aprendizado de máquina através de redes neurais e reconhecimento de padrões para desenvolver um algoritmo capaz de identificar plantas que contenham as doenças cancerígenas, cercosporiose e a ferrugem. Tinha como intuito partir deste diagnóstico fornecido pelo algoritmo, para possibilitar o agricultor tomar as medidas necessárias para tratamento de sua plantação evitando prejuízos futuros.

[de Sousa et al. 2019] realizou um estudo por meio da implementação da rede neural RBF (Radial Basis Function), objetivando usá-la como ferramenta de apoio no diagnóstico da doença HLB (Huanglongbing) em um pomar de tangerinas poncã. A metodologia foi implementada com base em 29 amostras e 8 atributos de uma base de dados construída, com ênfase no treinamento e teste para classificação das amostras utilizadas. Foram feitos 10 treinamentos com os dados balanceados e normalizados utilizando validação cruzada (leave-onte-out).

[Ferreira et al. 2019] discutiram o uso da lógica fuzzy como mecanismo auxiliar na análise do tomateiro para detectar se o mesmo é portador ou poderá desenvolver a doença murcha de fusariun. A metodologia baseou-se em pesquisas bibliográficas e em dados coletados em uma propriedade rural do interior do estado do Ceará. Os resultados demonstraram que o sistema fuzzy é bastante útil para o auxílio na detecção da doença evidenciando a importância da lógica nebulosa para diagnósticos em plantas.

\section{Redes Neurais Artificiais}

As Redes Neurais Artificiais (RNAs) são baseadas no funcionamento do cérebro humano e sua capacidade de aprendizagem. O processo de aprendizagem é realizado através de 
algoritmos de treinamento que ajustam os parâmetros livres da rede de forma a alcançar uma determinada resposta desejada. O primeiro modelo de neurônio artificial foi introduzido por McCulloch e Pitts em 1943 [Reis 2014].

Segundo [Fanucchi et al. 2013] existem diversas topologias de RNAs de forma a possibilitar a resolução de diversos tipos de problemas, tais como: Aproximação de funções, classificação de padrões, controle de processos, entre outros.

\subsection{Radial Basis Function (RBF)}

As redes do tipo Radial Basis Function (RBF) possuem apenas uma camada escondida e uma camada de saída. Possui também uma arquitetura do tipo forward, sendo que o sinal é propagado da entrada para a camada intermediária, que possui uma função de ativação do tipo gaussiana, e desta para a camada de saída, com uma função de ativação do tipo linear [Fanucchi et al. 2013]. A Figura 2 ilustra a estrutura básica de uma rede RBF

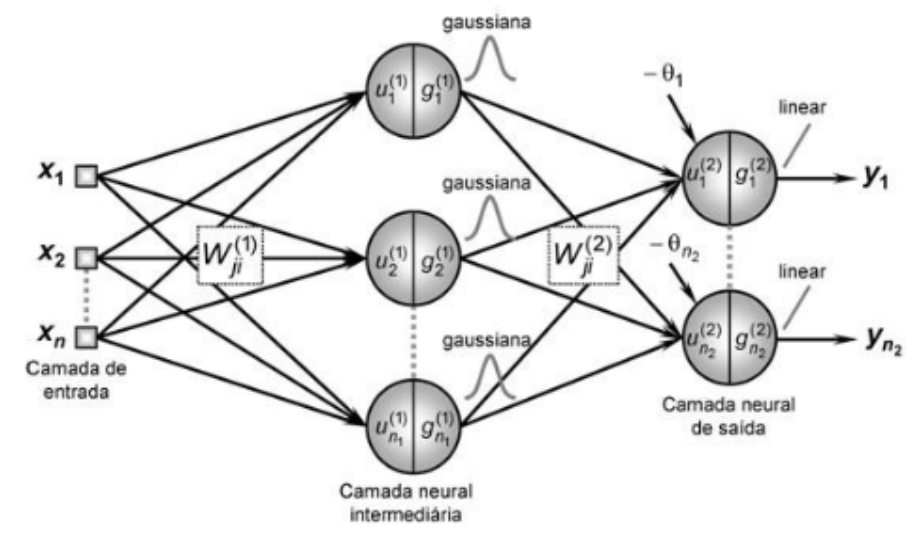

Figura 1. Arquitetura da Rede Neural RBF

\section{O Patógeno Phytophthora Capsici Leonian (PCL)}

A murcha de fitóftora ocorreu pela primeira vez no Brasil na região de Ribeirão Preto, São Paulo, em 1951. Causada pelo fungo PCL, tem sido uma das doenças mais destrutivas da cultura do pimentão desde então [Santos 2001].

\subsection{Sintomas}

O patógeno afeta primeiramente o sistema radicular e o colo da planta, podendo atacar a parte aérea. A PCL é um patógeno polífago, amplamente distribuído nos solos cultivados do Brasil e de muitos outros países, sendo de difícil controle [Lopes and de Ávila 2005]. A Figura 2 mostra comparativos entre plantas saudáveis e infectadas.

Os sintomas podem ser observados principalmente nas horas mais quentes do dia. Na região do coleto e das raízes das plantas observa-se uma podridão úmida, posteriormente estas plantas secam e morrem. Dependendo do estado de desenvolvimento, a planta infectada tem a possibilidade de não secar, mas seus frutos poderão murchar. Quando a umidade é muito elevada, o fungo pode desenvolver um micélio de coloração branca sobre ramos e frutos infectados ou produzir manchas encharcadas nas folhas, ramos e frutos [Pereira et al. 2013]. 


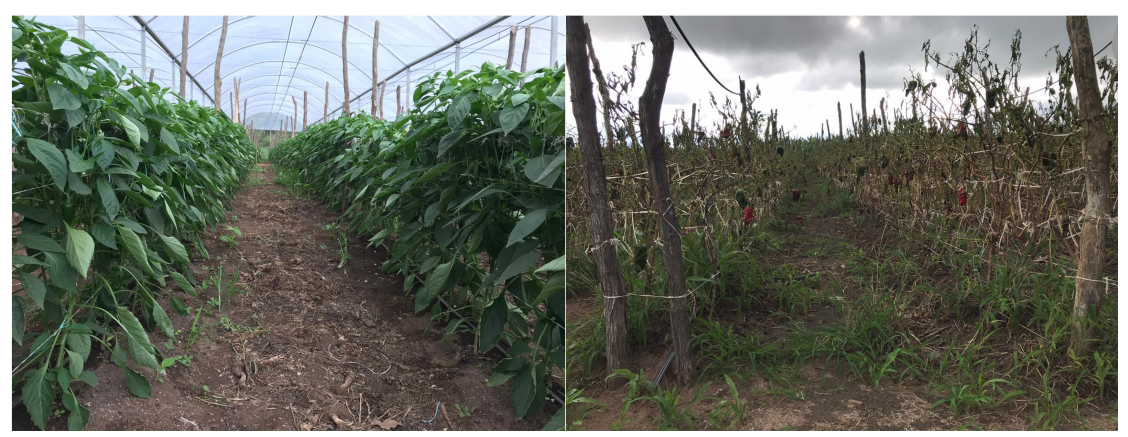

Figura 2. Comparativo Entre Plantas Saudáveis e Doentes

\subsection{Meios de Transmissão}

$\mathrm{Na}$ ausência do hospedeiro, o fungo passa a sobreviver saprofíticamente em restos de culturas ou fica dormente no solo, através dos oósporos, em condições favoráveis e na presença do hospedeiro germinam e infectam a planta [Santos 2001].

Normalmente os fungos produzem esporos ou fragmentos de hifas que são espalhados pelo vento ou por respingos de água de chuva ou de irrigação. Outras estruturas como clamidósporos, escleródios e oósporos, permitem a disseminação e sobrevivência de diversas espécies de fungos na ausência da planta hospedeira [Lopes and de Ávila 2005].

O fungo causador da doença reproduz-se assexuadamente através de esporângios que são dispostos em esporangióforos simpodiais, frequentemente elipsoides [Santos 2001]. Epidemias da podridão de frutos são mais comuns em períodos quentes $\left(20^{\circ} \mathrm{C}\right.$ a $\left.30^{\circ} \mathrm{C}\right)$ e chuvosos, em áreas irrigadas e em terrenos de baixadas, planos e mal drenados [Pereira and Pinheiro 2014]. O fungo penetra nas plantas pelas aberturas naturais, como estômatos e hidatódios, ou por meio de ferimentos [Viana et al. 2007].

O PCL é um oomiceto habitante de solo que sobrevive no solo e em restos culturais na forma de micélio e oósporos, estruturas resistentes à dessecação e condições ambientais adversas, que podem sobreviver no solo na ausência de hospedeiras por vários anos [Pereira and Pinheiro 2014].

\section{Metodologia}

Esta seção descreve a seguinte abordagem: (i) Base de dados utilizada; (ii) treinamento e testes realizados. Para o trabalho escolheu-se a rede neural RBF por apresenta-se com um modelo simples, com apenas uma camada oculta constituída por funções de ativação de base radial e uma camada de entrada associada diretamente as informações da entrada da rede [Reis 2014].

\subsection{Base de Dados}

Os dados foram coletados em uma horta de pimentões, na localidade Sítio Córrego, na cidade de Tianguá-CE, pelos autores. A base de dados possui um total de 127 amostras, compostas por 6 atributos, que foram divididos em 3 grupos. Cada grupo de características separadas por partes da planta como caule, folhas e frutos. Do caule foram coletadas informações como escurecimento na coroa das raízes no colo, próximo ao solo, 
e mofo branco. Já das folhas foram consideradas as características, necrose ou murchamento e lesões circulares com margens bronzeadas e centros castanhos. Por fim do fruto, apodrecimento do fruto ainda conectado a planta e mofo branco.

\subsection{Treinamentos e Testes}

Para o treinamento e teste utilizou-se a arquitetura da rede neural RBF, composta por 6 neurônios de entrada, uma camada oculta com 10 neurônios e uma camada de saída com 2 neurônios. Utilizou-se do algoritmo de redes neurais $R B F C l a s s i f i e r$, pertencente (Waikato Enviroment for Knowledge Analysis) (WEKA) suíte de mineração de dados muito popular no meio acadêmico, desenvolvida utilizando a linguagem Java. Criada nas dependências da Universidade de Waikato, Nova Zelândia. Atualmente mantida por uma comunidade de entusiastas por ser um software livre e disponível sobre a licença GPL [Damasceno 2015].

A base de dados foi normalizada e balanceada garantindo a proporcionalidade entre as amostras utilizadas. Para o balanceamento utilizou-se da ferramenta WEKA através do filtro de instância ClassBalancer que garantiu através da criação de dados sintéticos a equidade entre as amostras.

\section{Resultados dos testes}

Os resultados obtidos e analisados tiveram como base os percentuais de acurácia adquiridos nos treinamentos e testes realizados com a rede neural RBF assim como na discussão dos resultados encontrados por meio das amostras do banco de dados coletado.

Vale salientar que para o treinamento e teste foi utilizado o método de validação cruzada $k$-fold, com o $\mathrm{k}=10$ folds, testando também outras configurações com $\mathrm{k}=5, \mathrm{k}=$ $15, \mathrm{k}=20, \mathrm{~K}=25$ com a base de dados normalizada e balanceada.

Como observado na Tabela 1, o melhor caso obteve uma acurácia de 96,3\% de acertos e $3,7 \%$ de erros e o pior caso obteve $91,8 \%$ de acertos e $8,2 \%$ de erros. Deve-se salientar que a taxa média de acertos foi de $93,3 \%$ e $6,7 \%$ de erros.

Tabela 1. Resultados dos Testes Realizados com a Rede Neural RBF

\begin{tabular}{llcc}
\hline Treinamento & Taxa de Acerto (\%) & Taxa de erro (\%) & Situação resultado \\
\hline 1 & 92,6 & 7,4 & \\
\hline 2 & 96,3 & 3,7 & Melhor Caso \\
\hline 3 & 92,1 & 7,9 & \\
\hline 4 & 91,8 & 8,2 & Pior Caso \\
\hline 5 & 94,2 & 5,8 & \\
\hline 6 & 92,6 & 7,4 & \\
\hline 7 & 95,2 & 4,8 & \\
\hline 8 & 91,9 & 8,1 & \\
\hline 9 & 92,7 & 7,3 & \\
\hline 10 & 94,3 & 5,7 & Caso Médio \\
\hline Total & 93,3 & 6,7 & \\
\hline
\end{tabular}

Vale salientar que os resultados mostraram-se bastante satisfatórios com uma média de acerto acima de $90 \%$. 


\section{Conclusão e Trabalhos Futuros}

O artigo em questão trata sobre a utilização da rede neural artificial RBF como um mecanismo auxiliador no processo de pré-diagnóstico da doença Murcha de Fitóftora, com o intuito assim de ajudar profissionais da área da agricultura a tomarem as devidas providências para que os impactos poderiam ser gerados pela doença sejam minimizados e assim evitar grandes perdas de produção. Os resultados obtidos demonstraram-se significativos, já que conseguiram identificar quais plantas eram acometidas pela doença

Como trabalhos futuros sugere-se um estudo com outras redes neurais, utilizandoas como recurso auxiliar no diagnóstico da doença pinta preta em tomateiros, doença esta que provoca perdas significativas na produção gerando assim prejuízos econômicos aos produtores.

\section{Referências}

Castro, R. V. O. (2012). Modelagem de árvore individual para uma floresta estacional semidecidual utilizando redes neurais. Viçosa, $M G$.

Damasceno, M. (2015). Introdução a mineração de dados utilizando o weka. Disponivel: http://connepi. ifal. edu. br/ocs/index. php/connepi/CONNEPI2010/paper/viewFile/258/207, Acesso: setembro.

de Sousa, R., de Brito, R., and Ximenes, J. (2019). Aplicação da rede neural rbf como mecanismo auxiliador no diagnóstico da doença hlb em espécies citricas. In Anais da VII Escola Regional de Computação Aplicada à Saúde, pages 55-60, Porto Alegre, RS, Brasil. SBC.

Fanucchi, R. Z., Oleskovicz, M., and Barbosa, D. (2013). Análise da detecção de faltas de alta impedância utilizando redes neurais artificiais com topologias baseadas em perceptron multicamadas e redes rbf. Simpósio Brasileiro de Automação Inteligente (SBAI), Fortaleza.

Ferreira, A. F., Moreira, L., de Arruda, G., de Brito, R., and Ferreira, A. V. (2019). Sistema fuzzy como ferramenta auxiliar na detecção da murcha de fusarium no tomateiro. In Anais da VII Escola Regional de Computação Aplicada à Saúde, pages 193-198, Porto Alegre, RS, Brasil. SBC.

Galvão, G. F. P., Carvalho, W., Rocha, W., and da Silva Costa, J. C. (2018). Visão computacional para detecção de doenças fúngicas na agricultura. ÚNICA Cadernos Acadêmicos, 2(1).

Lopes, C. A. and de Ávila, A. (2005). Doenças do tomateiro. Embrapa Hortaliças-Livro técnico (INFOTECA-E).

Pereira, R., Pinheiro, J., and de Carvalho, A. (2013). Diagnose e controle alternativo de doenças em tomate, pimentão, curcurbitáceas e cenoura. Embrapa Hortaliças-Circular Técnica (INFOTECA-E).

Pereira, R. B. and Pinheiro, J. B. (2014). Phytophthora capsici em cucurbitáceas. Embrapa Hortaliças-Comunicado Técnico (INFOTECA-E).

Reis, F. A. (2014). Procedimento de ajuste de parâmetros de redes rbf via pso. 
Santos, H. S. (2001). Enxertia em plantas de pimentão (capsicum annuum 1.) no controle da murcha de fitóftora (phytophthora capsici) em ambiente protegido.

Viana, F., FREIRE, F., and Parente, G. (2007). Controle das principais doenças do pimentão cultivado nas regiões serranas do estado do ceará. Embrapa Agroindústria Tropical-Comunicado Técnico (INFOTECA-E). 